\title{
Technique Analysis of Dots-and-Boxes
}

\author{
Chenguang Zhang 1, a , Shuqin $\mathrm{Li}^{2, b}$, Xiaohua Yuan ${ }^{3, \mathrm{c}}$ \\ ${ }^{1}$ Beijing Information Science\& Technology University ,Beijing, P.R. China, \\ ${ }^{2}$ Beijing Information Science\& Technology University,Beijing, P.R. China \\ ${ }^{3}$ Shanghai Oceanic University, Shanghai, P.R.China \\ a 786763807@qq.com, bishuqin_de@126.com , c xhyuan@shou.edu.cn
}

\begin{abstract}
Keywords: Artificial intelligence; Dots-and-Boxes; chess board representation; equilibrium solution Abstract. As one important computer game, Dots-and-Boxes is always being taken as one project of the international computer Olympic tournament and the Chinese college students' computer game, and has already been listed in the 2015 Chinese intellectual games. In this paper, firstly, the rules and common terminology of Dots-and-Boxes was introduced, then the chess board representing method, the strategy influencing the game outcome, and the equilibrium solution to the chess state are analyzed. Our program adopting the strategies obtained 2015 Students Computer Game Championship.
\end{abstract}

\section{Introduction}

Computer game is an important research subject of artificial intelligence, and the development of artificial intelligence itself mainly profit from the development of computer game researches, in which, the notable events in 1997 of Deep Blue beat Garry Kasparov, the world chess champion, became one important milestone [1]. Invented in 1891 by Edward Lucas, one French mathematician, Dots-and-Boxes[2] is a two player game that using paper and pen. At earlier time, this game is very popular in European, being taken as the project of international computer Olympic tournament game. By now, many well-known international institutions have taken part in the development of related software for Dots-and-Boxes, for example, Elmo Timoteus with the Mathematics Department of UCLA (University of California at Los Angeles) has developed one software of Dots-and-Boxes, which not only of high efficiency, but also can adjust the board size at any time during the game [2]. In recent years, with the development of Chinese computer game championship, Dots-and-Boxes has gradually been known by the computer game enthusiasts. By now, Dots-and-Boxes has been listed as one of the major computer competition game, and it also is the game has the most participator number.

At present, only few articles has comprehensive introduce Dots-and-Boxes (fro details please refer to [3-6]). This paper will mainly introduce the basic concept of Dots-and-Boxes, study the board representation and winning factor and etc.

\section{Brief introduction of Game rules and basic concepts}

Dots-and-Boxes is a typical category of Tim games, at present, in the international computer Olympic tournament and the national college computer game competition, the board of $6 \times 6$, as shown in Figure 1, is adopted. 


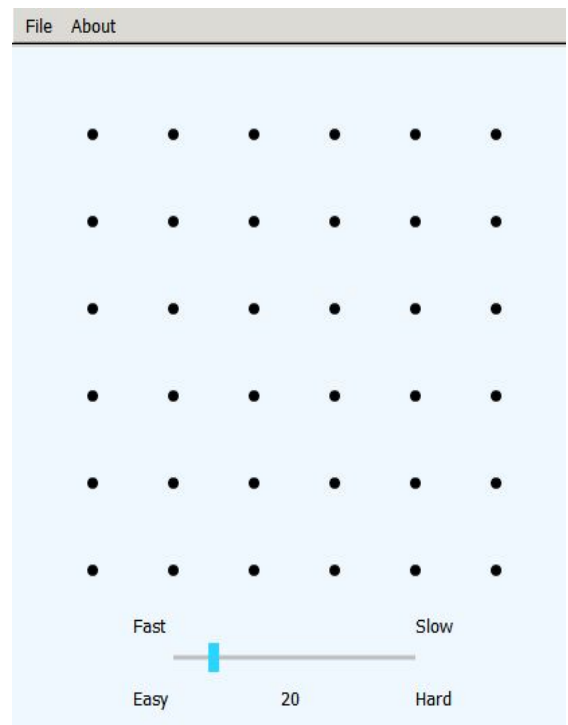

Fig. 1 Chess board before the game

\subsection{Game rules}

The game rules of Dots-and-Boxes are that.

(1) The sides make move alternately. When one side moves, it connects one line between the two dots near the side, abide by the limitation of that: the line cannot cross any dot, between two dots there only allows one line, and there also not allow any diagonal line.

(2) Every box is surrounded by four sides, and the owner of the last edge is the owner of the box.

(3) After owning the box by adding the last line, the winning side can further move, until there is no more unoccupied box.

(4) After all the boxes are occupied, the game ends, and the side owning larger number of box wins.

Below the Figure 1 to 3 are three main interfaces during the game. From Figure 3, we can see that at the end, the sides owning blue boxes win.

Generally, for the sake of fair, each competition will run two times, thus each side will have chance been the first player or the second player, since being the first or the second will have the different advantages.

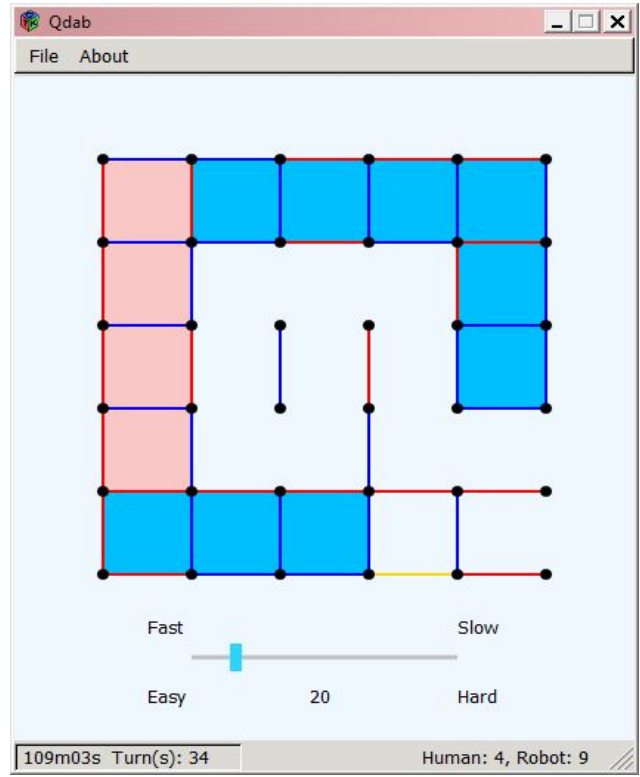

Fig. 2 Chess board during the game

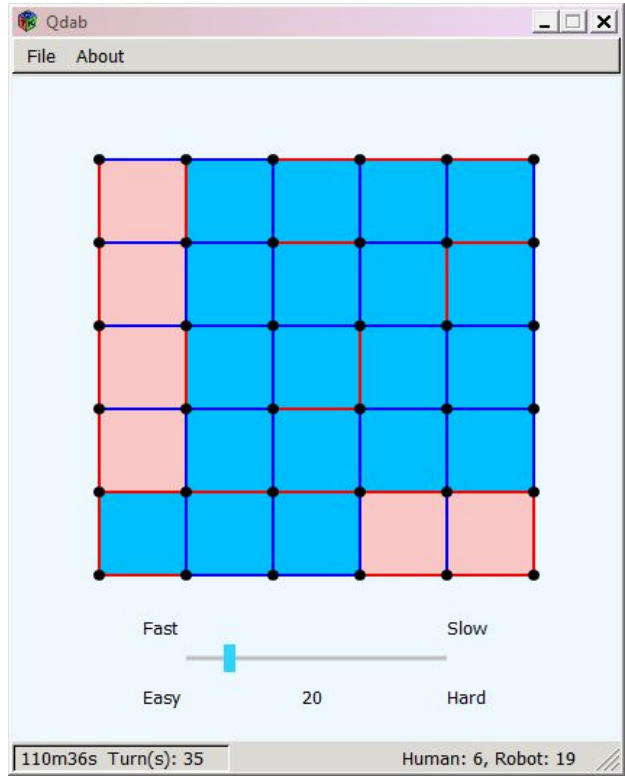

Fig.3 Chess board at game end 


\subsection{Basic concepts of Dots-and-Boxes}

In the studies of Dots-and-Boxes, some basic concepts are always involved in, which are.

(1) Degree of freedom. It is the number of unoccupied edges of one box.

(2) C type box, $\mathrm{C}$ type box is the box whose freedom degree is 1 .

(3) Adjacent, refer two boxes of location $(i, j)$ and $(k, 1)$, only if $|i-k|+|j-1|=1$, and the public side is not occupied.

(4) One turn, refer to that, after one side moved, the other side start to move.

(5) One move, refer to connect the line between two adjacent dots.

(6) Filling the box, refer to the move that close one box.

(7) Chain. If there a set of boxes $B N=\{b(i)\}, 0<=i<=n$, and each $b(i)$ has freedom degree 2 (that is , this box has unoccupied edges), and of $b(0)$ and $b(n)$, there is at most only one box belong to BS, and the left will has two adjacent boxes, is the adjacent boxes are also belong to BN, then BN is one Chain. Clearly, $b(0)$ and $b(n)$ are the terminal of the Chain, and they can be the same box.

Chain is divided into long one and short one. In which.

(1) Short chain, Short chain are Chain only consist of 1 or two boxes.

(2) Long chain, Long chain are Chain consist of three or more than three boxes

(8) Circle, means one chain $\mathrm{BN}=\{\mathrm{b}(\mathrm{i})\}, 0<=\mathrm{i}<=\mathrm{n}$, each $\mathrm{b}(\mathrm{i})$ has freedom degree 2 and has two adjacent box, and the two adjacent belongs to $\mathrm{BN}$ also, then $\mathrm{BN}$ is a Circle. A Circle is generally a long Chain, whose beginning and end box is the same box, so in a Circle there are at least four boxes.

(9) Double cross, means that the next move will occupy two boxes.

(10) The Final phase, refer to the phase that when there are only long Chain.

(11) Joint, refer to the boxes whose degrees of freedom is 3.

\section{Representation method of chess board}

In college computer game, the game of Dots-and-Boxes is time limited, with a predefined overall time. So the player must make full use of the time, to complete as much as possible searches and cost as less as possible time. Motivated by this, there are three types of Board representation.

(1) Board representation using coordinates

The traditional board representation take chess boar as one two-dimensional table, the position of each dot is represented by a pair of coordinates, further, the position of the boxes and the edges are similarly represented by their coordinates. Using coordinate representation is very convenient for interface designing, but it will induce much tedious in game searches.

(2) Board representation using digital number

Another board representation donates each dot with a number, and record its related coordinates, after deciding the next dot go to move, the number of the dot is transformed into the related coordinates, and the dot is shown at its position in the interface. Thus, it is not only relatively convenient in representing the edge, but the related searches are relative simple, and it has a great help to improve the efficiency of the game. Although using digital number to describe the board can reduce some of the tedious work in the representation of dot, there needs to searches the Chains of each dot, and to calculate the special position and relationship through coordinate, which need a complex search process, thus will bring great trouble to the distinguishing and constructing of Chain and Circle.

(3) Representation of box using point

In order to quickly determine the freedom degree of the long Chains, the Rings and the boxes, the other representation method use a point to express each box. Taking the idea of graph theory, the degree using in graph is used to represent the freedom degree of each box. The freedom degree of each point is initialized as 4, as shown in Figure 4, during the game, if some edges are formed, then short the connection between points, and the freedom degree of each dot minus 1 . This representation can conveniently record the feature values in the game, can reduce the time cost of search and the code amount, thus make the program process relative simple and clearly. 


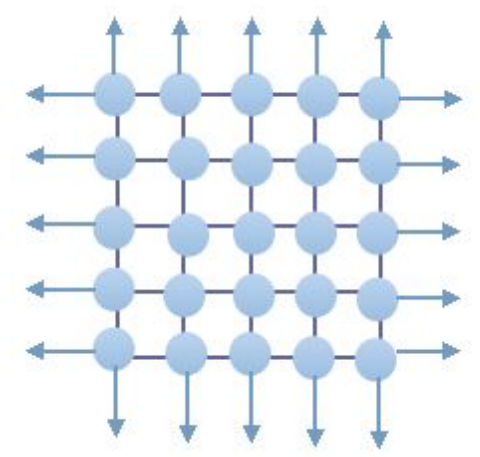

Fig.4 Chess board representation using point

\section{Analysis on main technical in Dots-and-Boxes}

\subsection{Two important strategies influencing the outcome}

According to the game rules of Dots and Boxes, if the player in his current turn can occupy the box, then after he occupied the box, he will continues to occupy some edge, until the edge lost the box. And according to the rules, the player who occupies the last long Chain is more likely to win, since he has taken the initiative in the winning of the former chain, and made the right selection from which he was befitted. Therefore, the strategies should be adopted in the competition are that.

Strategy 1: If the total number of the point in the board is odd, then the first hand should form an odd number long Chains, ad the second hand should form an even number long Chains. If total number of the point is even, then the first hand should form an even number of long Chains, and the second hand should form an odd long Chains.

In order to gain the initiative in the game, the side which has first entered one long Chain, who will be allowed two choices, one is to take the greedy algorithm, as possible as to occupy more boxes. Another is the striking method, that is, in the connecting of the last edge, try to give up four or two boxes, thus make the player himself own more boxes than the rival.

Strategy 2: In the end game, if there are more than one Circles and Chains coexisting, then count the numbers cc_box_num of the boxes closed by the Chains and Circles, here $\mathrm{n}$ denotes the number of Chains, and $m$ that of Circles. If cc_box_num $-8 m-4 n+4<0$, then chose the greedy algorithm, otherwise, select the striking method.

The player who takes the initiative, will form the last edge of each turn into one Circle if there is, otherwise, he will form this edge in one Chain, because if formed the edge in a Circle in the next turn he can obtain 4 boxes, and form the edge in a Circle can only obtain 2 boxes.

\subsection{Equilibrium solution in Dots-and-Boxes}

The board size of Dots-and-Boxes can be changed,we can set a rand board of size ${ }^{\mathrm{n} \times \mathrm{m}}$, where $\mathrm{n}$ and $\mathrm{m}$ means the dots number row-wise and column-wise, respectively, and the usually used are the $3 \times 3$ or ${ }^{6 \times 6}$ board. In the ${ }^{6 \times 6}$ board, there are 25 boxes, so there must be one winner and one loser. The purpose of both side is try to inhibit the opponent and to get more benefits. Since both sides are rational, so the best result is that the absolute difference between the box numbers of the two sides is minimized, thus this is a typical two player zero-sum game, that is, the sum of benefits obtained by the two sides are the zeros, the income of $A$ is just the outcome of $B$, and vice verse. Since we only analyze the solution of Dots-and-Boxes, so here we only consider the final phase of the board. The two player zero-sum game is usually described by matrix, like this, if A occupied the boxes of $\{0,1,2, \ldots, 25\}$, then B will own the boxes of $\{25,24,23, \ldots, 0\}$, the difference between the boxes occupied by $\mathrm{A}$ and $\mathrm{B}$ is used to represent the income. So, there is

$$
\mathrm{U}=\operatorname{diag}\{-25,-23, \ldots,-1,1, \ldots, 23,25\}
$$

where 0 means the state that can not arrived at. According to the method of minimax, there is 


$$
\begin{gathered}
\max _{i} \min _{j} u_{i j}=\max \{-25,-23, \ldots,-1\}=-1 \\
\min _{j} \operatorname{maxu}_{i j}=\min \{1,3, \ldots, 21,23,25\}=1
\end{gathered}
$$

Since $\max _{\mathrm{i}} \min _{\mathrm{j}} \mathrm{u}_{\mathrm{ij}} \neq \min _{\mathrm{j}} \max _{\mathrm{i}} \mathrm{u}_{\mathrm{ij}}$, it is not equilibrium state.

\section{Conclusion}

As one crystallization of human wisdom, intelligence movements of chess and cards have attracted many a few participators, by its special cultural attraction, and its profound cultural heritage and educational function have also been widely appreciated by current society. As the competition project of the International Computer Olympic tournament and of the Chinese college students' computer game, it was listed in the Chinese intellectual sports meeting in 2015.

This paper has introduced the origin, the basic concept, the board, as well as the winning factors of Dots-and-Boxes, which will give some lights for the future researches and studies. Dots-and-Boxes the 2nd National Intelligence Sports Meeting

\section{Acknowledgements}

This paper is jointly sponsored by the Education and Teaching Project for undergraduate scientific research plan of Education Committee of the Beijing Municipality. (PXM2015_014224_000050), by Opening Project of Beijing Key Laboratory of Internet Culture and Digital Dissemination Research (ICDD201507).

\section{References}

[1] Feng-Hsiung Hsu . Behind Deep Blue: Building the computer that defeated the world chess champion.. Princeton University Press; Revised edition. 2004, 239-245.

[2] Elmo, Timoteus .Dots and Boxes. Loc Publishing, 2011-07-30.

[3] Albert L. Zobrist. Feature Extraction and Representation for Pattern Recognition and the Game of Go. Ph.D. Dissertation, University of Wisconsin, 1970.

[4] Zhiqing Liu, Qing Dou. Automatic Pattern Acquisition from Game Records in GO. in the Journal of China University of Posts and Telecommunications. 2007 14(1): 100-105.

[5] M. Boon. A pattern marcher for Goliath. Computer Go. 1990, (13): 13-22.

[6] B. Bouzy, T. Cazenave. Computer Go: an AI oriented survey. Artificial Intelligence. 2001, 132(1): 39-103. 